Revista de

Contabilidade e Organizações www.rco.usp.br
DOI: http://dx.doi.org/10.11606/issn.1982-6486.rco.2020.168631
Journal of

Accounting and

Organizations

www.rco.usp.br

\title{
Impacto da evidenciação de informações no valor das ações das sociedades de economia mista
}

Impact of information disclosure on the value of shares of mixed economy companies

Octavio Locatelli ${ }^{a}$, Valcemiro Nossa ${ }^{\text {a }}$, Felipe Ramos Ferreira ${ }^{\mathrm{a}}$

a Fucape Business School

Palavras-chave

Teoria da Divulgação.

Disclosure obrigatório.

Disclosure voluntário.

Transferência informacional.

Sociedades de economia mista.
Keywords

Disclosure Theory.

Mandatory disclosure.

Voluntary disclosure.

Informational transfer.

Mixed economy societies.

\section{Resumo}

O objetivo desta pesquisa é verificar se a divulgação: (i) das demonstrações contábeis; (ii) dos comunicados ao mercado e; (iii) da classificação de rating do governo, afetam o valor das ações das sociedades anônimas de capital aberto. Adicionalmente, a pesquisa, também, tem como objetivo identificar se o impacto nas sociedades de economia mista ocorre em maior grau quando comparadas as demais firmas. A pesquisa tem como base temporal o intervalo de tempo de 2003 a 2015, e a amostra é composta por 3.514 observações, empresa/ano, sendo 239 observações de sociedades de economia mista. Os eventos de divulgação de demonstrações contábeis e de comunicados ao mercado foram coletados manualmente no site da CVM e os eventos de rating soberano, no site da Secretaria do Tesouro Nacional. Foram realizadas regressões múltiplas, que demonstraram que os retornos anormais acumulados são impactados pelas divulgações obrigatórias, voluntárias e pelas classificações de rating do governo. Além disso, observou-se que há um impacto adicional nas empresas de economia mista quando das divulgações voluntárias e de classificação de rating soberano.
Informações do artigo

Recebido: 09 de abril de 2020

Aprovado: 31 de agosto de 2020

Publicado: 05 de outubro de 2020

\section{Implicações práticas}

A pesquisa contribui para uma melhor compreensão do mercado de capitais brasileiro e seus investidores, mostrando a reação destes à divulgação de informações das empresas. Além disso, mostra que os investidores reagem de forma adicional quando a divulgação de informações é sobre empresas que tem o governo como acionista. 


\section{INTRODUÇÃO}

O propósito da Teoria da Divulgação é explicar os fenômenos decorrentes da divulgação de informações financeiras (Salotti \& Yamamoto, 2005). Uma das perspectivas estudadas é o impacto da divulgação de informações no valor das ações das companhias. Para alguns autores como Gibbins, Richardson e Waterhouse (1990) e Von Alberti-Alhtaybat, Hutaibat e Al-Htaybat (2012), o disclosure corporativo é um termo de grande alcance, tratando da evidenciação de informações, financeiras ou não, numéricas ou qualitativas, obrigatórias ou voluntárias, por meio físico ou digital. Além disso, pode ser integrado a essa definição, a divulgação de informações de forma involuntária (Skillius \& Wennberg, 1998). Por outro lado, Verrecchia (2001) afirma não haver uma Teoria da Divulgação unificada ou integrada, visto que não existe um paradigma central que dê base a todas as pesquisas, mas que essas examinam pequenas partes do todo.

Healy e Palepu (2001) discorrem que as organizações podem divulgar informações obrigatórias, por exemplo, por intermédio dos relatórios financeiros, voluntárias, por meio de comunicados na imprensa e relatórios corporativos e, involuntárias, por meio de analistas financeiros e da imprensa. Eles afirmam ainda que essa divulgação é essencial para que o mercado de capitais funcione eficientemente.

No Brasil, as sociedades de economia mista (SEM) são agentes econômicos de relevância e se apresentam em número expressivo, visto que representam uma das formas de atuação do governo na economia (Ribeiro \& Alves, 2006). Porém, além dos diversos problemas de ineficiência do governo em setores vitais ao desenvolvimento do país, as sociedades de economia mista sofrem os riscos de influência política, a qual é capaz de afetar o valor das empresas que o governo figura como investidor (Kuschbrey et al., 2014). Soma-se a esse contexto o fato de que o valor das ações de uma empresa pode ser afetado pelo seu disclosure e pelo disclosure de terceiros, como visto nas pesquisas de transferência informacional.

Assim, essa pesquisa tem por objetivo analisar se as divulgações de informações obrigatórias e voluntárias sobre a firma, bem como a divulgação de informações sobre o governo afetam o preço das ações das empresas. Adicionalmente, é analisado se a divulgação dessas informações afeta diferentemente as sociedades de economia mista.

A justificativa desta pesquisa está na importância de uma melhor compreensão do mercado de ações brasileiro, do comportamento das ações e reação dos investidores à divulgação de informações. Além de verificar a relevância e a funcionalidade da contabilidade, dos comunicados ao mercado e das classificações de rating soberano como redutores da assimetria de informações entre empresas e investidores. Cumpre destacar que o Brasil se torna um cenário propício para tal análise devido à forte participação do governo como investidor de grandes empresas no mercado acionário brasileiro.

Para tanto foram analisados os dados de 383 empresas listadas na B3 entre os anos de 2003 a 2015. Mais especificamente foram calculados os retornos anormais das firmas em torno dos eventos de divulgação de informações obrigatórias, como as Demonstrações Financeiras Padronizadas (DPF) e Informações Trimestrais (ITR), voluntárias, como os comunicados ao mercado, e de transferência informacional, como as divulgações de rating soberano. Os resultados evidenciam que as sociedades de economia mista bem como as demais empresas são afetadas igualmente no que se refere ao disclosure obrigatório. Entretanto, no que tange aos disclosure voluntários e do rating soberano as sociedades de economia mista apresentam um efeito adicional quando comparada as demais empresas.

Este estudo contribuiu em pelo menos dois aspectos para a literatura de contabilidade e finanças. Primeiramente, amplia a discussão sobre o efeito das informações divulgadas pela empresa sobre o preço de suas ações, mostrando evidências empíricas no mercado acionário brasileiro. Em segundo lugar contribui para debates sobre transferência informacional relacionada a um importante shareholder no mercado de ações brasileiro, o governo. Adicionalmente, esta pesquisa contribui para aspectos práticos, pois demonstra como o mercado se comporta diante da divulgação de informações empresariais e de rating soberano, permitindo uma maior compreensão por parte dos investidores sobre eventos de disclosure no cenário brasileiro. 


\section{REFERENCIAL TEÓRICO}

\subsection{Disclosure obrigatório}

Segundo Skillius e Wennberg (1998) o disclosure obrigatório é a evidência de informações sobre as atividades da companhia que é exigida por lei e independe de qualquer relação entre custo e benefício para divulgar (Mapurunga, Ponte \& Holanda, 2014). Entre as pesquisas empíricas, pode-se destacar as que relacionam disclosure obrigatório à volatilidade, preço e retorno de ações.

Malaquias e Lemes (2015) analisaram a relação entre o nível de disclosure de relatórios financeiros e a volatilidade do retorno dos títulos de empresas brasileiras listadas na NYSE. Para isso, estudaram os relatórios contábeis de 24 empresas brasileiras que possuíam ADRs níveis II ou III na NYSE, no período de 2002 a 2006 . E encontraram que quanto maior o nível de disclosure menor a volatilidade dos retornos de seus títulos, além disso, eles verificaram também que há relação entre o nível de disclosure e o tamanho das companhias.

Mugaloglu e Erdag (2011) estudaram o index ISE-30 após a implantação da Istanbul Stock Exchange Public Disclosure Platform, e de forma contrária a Malaquias e Lemes (2015), encontraram que para algumas empresas não há impacto na volatilidade e para outras a relação é positiva, mostrando que a expectativa de menor incerteza e volatilidade após a implantação da plataforma não se concretizou.

Já Sadeghzadeh e Karimi (2010) investigaram o impacto do disclosure obrigatório e voluntário no valor das ações de empresas do Tehran Stock Exchange. Para isso, estudaram 91 companhias nos anos de 2002 a 2009 , utilizando um checklist e o índice de Jensen. E observaram que nesse mercado, tanto o disclosure obrigatório quanto o voluntário não influenciam os investidores na tomada de decisão.

Também sem encontrar relação significativa, Marques et al. (2010) verificaram se o valor das ações de companhias participantes do Novo Mercado da BOVESPA é impactado pela publicação de Fatos Relevantes. Para realizar a pesquisa procederam um estudo de eventos com 92 publicações de fatos relevantes de 20 empresas do Novo Mercado. E como resultados observaram que a maioria dos fatos relevantes não influenciaram significativamente no preço das ações.

Partindo dos conceitos de disclosure obrigatório e tendo em vista a Lei 6.404/1976 que obriga a divulgação das demonstrações financeiras, bem como baseando-se nos estudos que relacionam a divulgação das demonstrações financeiras ao valor das ações, essa pesquisa tem como primeira hipótese: capital aberto.

$\mathbf{H}_{1}$ : O disclosure obrigatório das demonstrações financeiras impacta o valor das ações das companhias de

\subsection{Disclosure voluntário}

O disclosure voluntário pode ser definido como a divulgação de informações pelas empresas de forma discricionária (Skillius \& Wennberg, 1998), tendo por base a relação entre custo e benefício para divulgar (Mapurunga, Ponte \& Holanda, 2014). De forma semelhante, Dye (2001) trata o disclosure voluntário como um caso particular da Teoria dos Jogos, no qual a empresa divulgaria as informações se essas fossem benéficas para ela. Adicionalmente, Sousa et al. (2014) afirmam que as organizações divulgam informações de forma voluntária para se diferenciarem no mercado, orientando os investidores a tomar decisões que maximizem o valor das mesmas.

Murcia et al. (2011) pesquisaram se o nível de disclosure impacta na volatilidade das ações de companhias abertas no Brasil. Para isso fizeram uma análise de conteúdo, de divulgação voluntária, nas Demonstrações Financeiras Padronizadas das 100 maiores empresas da BOVESPA, excetuando-se as financeiras. E identificaram que a volatilidade das ações é impactada pelo disclosure econômico, porém o disclosure socioambiental e o disclosure total não apresentaram significância para alterar a volatilidade das ações.

Sousa et al. (2014) estudaram as empresas listadas na BOVESPA, também classificando o disclosure em social, ambiental e econômico. Para isso fizeram uma análise das Demonstrações Financeiras e dos Relatórios Anuais e de Sustentabilidade de 97 empresas, no período de 2007 a 2011. E encontraram como resultados que há relação positiva entre disclosure econômico e valor de mercado, há relação negativa entre disclosure ambiental e valor de mercado, e que essa relação não existe para o disclosure social.

Já Lima et al. (2012) realizaram um estudo experimental simulando um mercado de capitais com duas empresas e 353 participantes, na qual uma das empresas divulgava informações sobre o desempenho e a outra não. E trouxeram como conclusões que o nível de disclosure das organizações impacta as perspectivas dos investidores, e, por conseguinte, no valor das ações. 
Além disso, averiguaram também que a política de disclosure, dos dividendos e a estrutura de capital afetam não só o valor das ações da própria empresa como também das concorrentes que não fazem as mesmas divulgações.

A partir dos conceitos de disclosure voluntário, que se referem à divulgação de informações de forma voluntária (Skillius \& Wennberg, 1998; Mapurunga, Ponte \& Holanda, 2014; Sousa et al., 2014; Dye, 2001) e do Pronunciamento de Orientação CODIM 5, de 27 de novembro de 2008, que discorre, entre outros assuntos, sobre a divulgação facultativa de comunicados ao mercado, ergue-se a segunda hipótese desse estudo:

$\mathbf{H}_{2}$ : $\mathrm{O}$ disclosure voluntário, de comunicados ao mercado, impacta o valor das ações das sociedades anônimas de capital aberto.

\subsection{Disclosure involuntário e transferência informacional}

O disclosure involuntário é definido por Skillius e Wennberg (1998) como a exposição de informações sobre as atividades da empresa sem consentimento e de forma contrária a vontade da mesma. Destaca-se pesquisas que estudaram a relação entre divulgação involuntária e preço das ações utilizando a classificação de rating emitida pelas agências de risco. Murcia, Murcia e Borba (2013) analisaram se o rating de crédito de sociedades abertas brasileiras impacta no retorno das ações, por meio de 242 ratings anunciados pelas agências Standard \& Poor's e Moody's no período de 1997 a 2011. E encontraram resultados significativos para retornos anormais em todos os tipos de anúncio de rating, inicial, downgrades e upgrades, evidenciando assim que os anúncios de rating têm conteúdo informacional, pois afetam os retornos das ações.

Creighton, Gower e Richards (2007) também estudaram a reação dos preços das ações aos anúncios de mudanças de rating no mercado australiano. Para isso, realizaram um estudo de eventos com 141 anúncios emitidos pelas agências Standard \& Poor's e Moody’s entre 1990 e 2003. Encontrando uma relação entre os anúncios e a variação dos preços, porém o impacto verificado foi pequeno.

De forma parecida, Jorion, Liu e Shi (2005) realizaram um estudo de eventos com 1767 downgrades e 437 upgrades divulgados pelas agências Standard \& Poor's, Moody’s e Fitch. Como resultados encontraram relações significativas para downgrades no período pré-regulação e tanto para upgrades quanto para downgrades no período pós-regulação. Eles concluíram também que após a regulação as ações sofreram maior impacto.

Segundo Dietrich (1989) o principal conceito de transferência informacional é a noção de que os investidores interpretam as informações de uma empresa na avaliação de uma negociação não apenas da própria empresa, mas também de outras empresas. Dietrich (1989) apresenta também três motivações para os estudos de transferência informacional. A primeira se refere à incorporação das informações pelo mercado nos preços das empresas não divulgadoras, a segunda ao impacto de divulgação de informações específicas de uma empresa a outras empresas do setor, e a última motivação é o desenvolvimento de precificação de ativos considerando uma difusão das informações.

Estudando o impacto dos resultados de uma empresa sobre o valor das ações de empresas do mesmo setor Foster (1981) encontrou evidências que o impacto existe, mas tem maior peso em empresas que têm a maior parte de suas receitas na mesma linha de negócios. Schneible Jr (2015) além de verificar que há transferência informacional no anúncio de ganhos, observou que quanto menor a distância estratégica entre as empresas mais positiva é essa transferência, e isso também ocorre quando a empresa anunciadora é maior e seus lucros mais persistentes.

Por meio dos conceitos de transferência informacional definidos por Dietrich (1989), das pesquisas de Foster (1981) e Schneible Jr (2015), que verificaram o impacto proveniente de transferências informacionais, das pesquisas de Murcia, Murcia e Borba (2013), Creighton, Gower e Richards (2007) e Jorion, Liu e Shi (2005), que relacionam rating a valor das ações e tendo em vista que a classificação de rating soberano não é uma informação diretamente relacionada às empresas, fundamenta-se a terceira hipótese:

$\mathbf{H}_{3 a}$ : A divulgação de classificação de rating soberano pelas agências de classificação de risco impacta o valor das ações das sociedades anônimas de capital aberto.

Ainda se utilizando dos conceitos e das pesquisas de transferência informacional e das pesquisas que relacionam classificação de rating a valor das ações, e levando em consideração a participação do governo no controle das sociedades de economia mista, tem-se uma última hipótese dessa pesquisa:

$\mathbf{H}_{3 \mathbf{b}}$ : A divulgação de classificação de rating soberano pelas agências de classificação de risco impacta de forma adicional o valor das ações das sociedades de economia mista. 


\section{METODOLOGIA DA PESQUISA}

O campo de estudo desta pesquisa são as empresas brasileiras listadas na B3, pois analisa o impacto no valor de suas ações pela evidenciação de informações. Além disso, compõe a amostra as sociedades de economia mista descritas na Tabela 1. Para os testes estatísticos, foi removido da amostra o Banco do Estado de Santa Catarina S.A. - Besc, por falta de observações das variáveis necessárias.

Tabela 1. Descrição da amostra

\begin{tabular}{|c|c|c|}
\hline Empresa & Setor & Principal acionista \\
\hline Banco da Amazônia S.A. & Finanças e Seguros & União Federal (Tesouro Nacional) \\
\hline Banco de Brasília S.A. - BRB & Finanças e Seguros & Governo do Distrito Federal \\
\hline Banco do Brasil S.A. & Finanças e Seguros & União Federal (Tesouro Nacional) \\
\hline Banco do Estado de Santa Catarina S.A. - Besc & Finanças e Seguros & União Federal (Tesouro Nacional) \\
\hline Banco do Estado de Sergipe S.A. - Banese & Finanças e Seguros & Governo do Estado de Sergipe \\
\hline Banco do Estado do Pará S.A. - Banpará & Finanças e Seguros & Estado do Pará \\
\hline Banco do Estado do Rio Grande do Sul S.A. - Banrisul & Finanças e Seguros & Estado do Rio Grande do Sul \\
\hline Banco do Nordeste do Brasil S.A. & Finanças e Seguros & União Federal (Tesouro Nacional) \\
\hline Banestes S.A. Banco do Estado do Espirito Santo & Finanças e Seguros & Governo do Estado do Espirito Santo \\
\hline Cemig Distribuição S.A. - Cemig & Energia Elétrica & Estado de Minas Gerais \\
\hline Centrais Elétricas Brasileiras S.A. - Eletrobrás & Energia Elétrica & União Federal (Tesouro Nacional) \\
\hline Cia Cat. de Águas e Saneamento - Casan & Outros & Estado de Santa Catarina \\
\hline Cia Saneamento Básico Est. São Paulo - Sabesp & Outros & Governo do Estado de São Paulo \\
\hline Cia. de Saneamento do Paraná - Sanepar & Outros & Estado do Paraná \\
\hline Companhia Celg de Participações - Celgpar & Energia Elétrica & Estado de Goiás \\
\hline Companhia de Saneamento de Minas Gerais - Copasa & Outros & Estado de Minas Gerais \\
\hline Companhia Energética de Brasília - Ceb & Energia Elétrica & $\begin{array}{l}\text { Secretaria de Estado de Fazenda do } \\
\text { Distrito Federal }\end{array}$ \\
\hline Companhia Energética de São Paulo - Cesp & Energia Elétrica & Governo do Estado de São Paulo \\
\hline Companhia Paranaense de Energia - Copel & Energia Elétrica & Estado do Paraná \\
\hline Petróleo Brasileiro S.A. - Petrobras & Petróleo e Gás & União Federal (Tesouro Nacional) \\
\hline São Paulo Turismo S. A. - SPTurismo & Outros & Pmsp Prefeitura Mun. São Paulo \\
\hline Telec. Brasileiras S.A. - Telebrás & Telecomunicações & União Federal (Tesouro Nacional) \\
\hline
\end{tabular}

Fonte: dados da pesquisa.

Os dados utilizados foram os momentos em que ocorreram os eventos de disclosure. Eles foram utilizados como marcos, de forma a definir os períodos de análise do valor das ações. As datas das divulgações das DFPs, ITRs e dos comunicados ao mercado foram coletadas manualmente no site da CVM e dos eventos de divulgação de rating soberano, emitidos pelas agências Standard \& Poor's, Fitch Ratings e Moody's, coletados no site da Secretaria do Tesouro Nacional.

O período analisado na pesquisa é do início do ano de 2003 ao final de 2015. Visto que 2003 é o início do período em que há dados referentes a comunicados ao mercado disponíveis para pesquisa no site da CVM. As variáveis analisadas são os retornos anormais das ações acumulados no período definido pelos eventos de disclosure. Quanto à janela de evento, período em que foram acumulados os retornos referentes a cada evento, que é abrangida por um nível de subjetividade (Camargos \& Barbosa, 2003), foi definida em 1 dia anterior à data do evento, a própria data do evento e 1 dia posterior, a exemplo de Beyer et al. (2010).

Para o cálculo dos retornos na pesquisa foi utilizada a forma de capitalização discreta, na qual os retornos das ações foram obtidos pela equação (1).

$$
r=\left(P_{t}-P_{(t-1)}\right) / P_{(t-1)}
$$

Em que $r$ é a taxa de retorno, $P_{t}$ o preço da ação no período $t$ e $P_{(t-1)}$ o preço da ação no período $t-1$.

Para os retornos anormais esta pesquisa utilizou o modelo de retorno ajustado ao mercado. No qual os retornos anormais são calculados pela diferença entre o retorno observado da firma e o retorno de um portfólio de mercado no mesmo período de tempo, conforme equação (2). 
No Brasil, o índice de mercado mais utilizado é o IBOVESPA (Camargos \& Barbosa, 2003), porém neste estudo se utilizou como retorno diário normal a média dos retornos (value-weighted return) diários de todas as empresas listadas na B3, ponderada pelo valor de mercado da empresa em relação ao total.

$$
A_{i t}=R_{i t}-R_{m t}
$$

Em que $A_{i t}$ é o retorno anormal de uma determinada ação $i$ no período de tempo $t, R_{i t}$ é o retorno observado da mesma ação $i$ no mesmo período de tempo $t$ e $R_{m t}$ é o retorno do portfólio de mercado no período $t$.

Para acumular os retornos anormais nos dias da janela de evento, visto que não há como precisar o momento em que o mercado recebeu as informações dos eventos (Camargos \& Barbosa, 2003) foi utilizado o Cumulative Abnormal Return (CAR) (3), que consiste em acumular os retornos anormais observados na janela de evento por meio de suas somas.

$$
\operatorname{CAR}_{i}\left(t_{1}, t_{2}\right)=\sum_{t=t_{1}}^{t_{2}} A_{i t}
$$

Em que $C A R_{i}$ é o retorno anormal acumulado do ativo $i, t_{1}$ é o primeiro dia da janela de evento, $t_{2}$ é o último dia da janela de evento e $A_{i t}$ é o retorno anormal de uma determinada ação $i$ no período de tempo $t$.

Para o teste estatístico, foi realizada uma regressão, semelhante à elaborada por Beyer et al. (2010):

$$
C A R_{i, t}=a+b_{1} \operatorname{car}_{i, t}^{D O}+b_{2} \operatorname{car}_{i, t}^{D V}+b_{3} \operatorname{car}_{i, t}^{G O V}+b_{4} V m_{i, t}+b_{5} \operatorname{Roa}_{i, t}+b_{6} \text { Btm }_{i, t} b_{7} I F R S_{t}+\varepsilon_{i, t}
$$

Em que $C A R_{i, t}$ é o retorno anormal acumulado do ativo $i$, no ano $t, c_{i, t}{ }^{D O}$ é o retorno anormal acumulado de três dias com centro nos anúncios de disclosure obrigatório das demonstrações contábeis, $c{ }_{i, t}{ }^{D V}$ é o retorno anormal acumulado de três dias com centro nos anúncios de disclosure voluntário de comunicados ao mercado e $c a r_{i, t}{ }^{G O V}$ é o retorno anormal acumulado de três dias com centro nos anúncios com transferência informacional de classificação de rating soberano do governo. Com o intuito de controlar para potenciais efeitos associados ao tamanho, rentabilidade e crescimento das empresas foram adicionadas ao modelo algumas variáveis de controle. A variável $V m$ é o logaritmo neperiano do valor de mercado da empresa $i$ no período $t$. A variável Roa, representa o retorno sobre o ativo da empresa $i$ no período $t$. O Book-to-Market da empresa $i$ no período $t$ é representado pela variável Btm. IFRS é uma dummy que vale 1 nos anos de 2010 e posteriores e 0 caso contrário, para refletir possíveis impactos das alterações contábeis.

Nos casos em que não tenha ocorrido anúncio, as variáveis $c a r_{i, t}{ }^{D O}, c_{i, t}{ }^{D V}$ e $c a r_{i, t}{ }^{G O V}$ assumiram o valor 0 e nos casos que ocorreram mais de um evento, os retornos de cada janela foram somados.

Para identificar o impacto adicional que sofrem as sociedades de economia mista sobre as divulgações foram adicionadas ao modelo interações entre as variáveis e uma variável dummy que identifica essa classe de empresa.

$$
\begin{gathered}
C A R_{i, t}=a+b_{1} S E M_{i, t}+b_{2} \operatorname{car}_{i, t}^{D O}+b_{3} \operatorname{car}_{i, t}^{D O} \times S E M_{i, t}+b_{4} \operatorname{car}_{i, t}^{D V}+b_{5} \operatorname{car}_{i, t}^{D V} \times S E M_{i, t}+b_{6} \operatorname{car}_{i, t}^{G O V}+ \\
b_{7} \operatorname{car}_{i, t}^{G O V} \times S E M_{i, t}+b_{8} \operatorname{Vm}_{i, t}+b_{9} \operatorname{Roa}_{i, t}+b_{10} \operatorname{Btm}_{i, t}+b_{11} I F R S_{t}+\varepsilon_{i, t}
\end{gathered}
$$

Em que SEM é uma variável dummy que assume valor 1 se a empresa é uma sociedade de economia mista e 0 caso contrário. As demais variáveis seguem a mesma definição da equação (4).

\section{RESULTADOS}

A Tabela 2 expõe a estatística descritiva da amostra demonstrando a relevância das sociedades de economia mista por meio do teste de diferença de médias, visto que essas empresas apresentam significativamente maior média de Vm, Roa e Btm quando comparadas as demais empresas. 
Tabela 2. Estatística descritiva

\begin{tabular}{lcccc}
\hline & CAR $_{\mathrm{i}, \mathrm{t}}$ & $\mathbf{V m}$ & Roa & Btm \\
\hline Média Total & 9.64437 & $\mathrm{R} \$ 5.883 .423$ & -9.41813 & 0.10068 \\
Desvio Padrão & 46.26848 & $\mathrm{R} \$ 16.762 .351$ & 75.21577 & 5.23112 \\
Média SEM & 7.45374 & $\mathrm{R} \$ 15.821 .126,47$ & 1.15104 & 1.47645 \\
Média das demais empresas & 9.80423 & $\mathrm{R} \$ 5.113 .549$ & -10.20851 & -0.00610 \\
\hline Teste T & & & $-3,9379$ \\
$\mathrm{t}$ & 0,7581 & $-8,9755$ & $-2,2535$ & $-1,48255$ \\
diff & 2,35049 & -10707577 & $-11,35956$ & 0,0001 \\
$\operatorname{Pr}(|\mathrm{T}|>|\mathrm{t}|)$ & 0,4484 & 0,0000 & 0,0243 & \\
\hline
\end{tabular}

Fonte: dados da pesquisa.

Nota: Em que $C A R_{i, t}$ é o retorno anormal acumulado do ativo $i$, no ano $t, V m$ é o valor de mercado das empresas em reais, Roa é o retorno sobre o ativo e Btm é o Book-to-Market.

A Tabela 3 apresenta as correlações das variáveis de controle dos modelos e a variável dependente. Tendo mostrado correlação significativa entre a variável dependente e a variável Btm, entre a variável $V m$ e as variáveis Roa e Btm, e entre a variável Roa e Btm.

Tabela 3. Correlações das variáveis de controle

\begin{tabular}{cllll}
\hline & \multicolumn{1}{c}{ CAR $_{\mathrm{i}, \mathrm{t}}$} & \multicolumn{1}{c}{ Vm } & \multicolumn{1}{c}{ Roa } & \\
\hline CAR $_{\mathrm{i}, \mathrm{t}}$ & 1,0000 & & & \\
$\mathrm{Vm}$ & $-0,0290$ & 1,0000 & & \\
Roa & $-0,0181$ & $0,0676^{*}$ & 1,0000 & 1,0000 \\
Btm & $-0,1379^{*}$ & $0,0357^{*}$ & $0,4305^{*}$ & \\
\hline
\end{tabular}

Fonte: dados da pesquisa.

Nota: Em que $C A R_{i, t}$ é o retorno anormal acumulado do ativo $i$, no ano $t, V m$ é o valor de mercado das empresas em reais, Roa é o retorno sobre o ativo e Btm é o Book-to-Market. * significante a $10 \%$.

Para os testes estatísticos foram utilizadas 3514 observações, delineadas por empresa e por ano, sendo que dessas 239 observações são de sociedades de economia mista. Cumpre destacar que não há observações para todas as empresas/ano, e que há perdas de observações quando são adicionadas as variáveis de controle por limitação dos dados dessas variáveis.

As variáveis $C A R_{i, p} c a r_{i, t}{ }^{D O}, c_{i, t}{ }^{D V}$ e $c a r_{i, t}{ }^{G O V}, V m, R o a$ e $B t m$ passaram pelo processo de winsorização com limite inferior de $1 \%$ e superior de $99 \%$, que consiste em substituir os valores abaixo e acima do limite determinado pelos menores e maiores valores remanescentes, respectivamente. Cumpre destacar que os resultados se mantêm semelhantes sem o processo de winsorização.

A Tabela 4 apresenta as regressões entre as variáveis, nos modelos simples e com interações, além dos controles. O modelo 1 relaciona o retorno anormal acumulado do ano ao retorno anormal acumulado em torno dos eventos de divulgação obrigatória, de divulgação voluntária e de classificação de rating do governo. O modelo 2 acrescenta as quatro variáveis de controle. Nos modelos 3 e 4, além das variáveis relacionadas nos modelos 1 e 2, estão as interações dessas variáveis com a variável SEM, representando o valor adicional que as variáveis apresentam quando estão relacionadas as SEM (Tabela 4). Todos os modelos foram estimados utilizando erropadrão robusto para controle de heterocedasticidade. 
Tabela 3. Resultado das regressões

\begin{tabular}{|c|c|c|c|c|}
\hline $\mathrm{CAR}_{\mathrm{i}, \mathrm{t}}$ & Modelo 1 & Modelo 2 & Modelo 3 & Modelo 4 \\
\hline \multirow{2}{*}{ Intercepto } & 6,13710 & 30,77421 & 6,14645 & 31,29829 \\
\hline & $(0,000)^{* * *}$ & $(0,000)^{* * *}$ & $(0,000)^{* * *}$ & $(0,000)^{* * *}$ \\
\hline \multirow{2}{*}{$\mathrm{car}_{\mathrm{i}, \mathrm{t}}^{\mathrm{DO}}$} & 0,91072 & 0,9138611 & 0,92081 & 0,9300167 \\
\hline & $(0,000)^{* * *}$ & $(0,000)^{* * *}$ & $(0,000)^{* * *}$ & $(0,000)^{* * *}$ \\
\hline \multirow{2}{*}{$\mathrm{car}_{\mathrm{i}, \mathrm{t}}$ DV } & 0,71544 & 0,6943111 & 0,74946 & 0,7308305 \\
\hline & $(0,000)^{* * *}$ & $(0,000)^{* * *}$ & $(0,000)^{* * *}$ & $(0,000) * * *$ \\
\hline \multirow{2}{*}{$\mathrm{car}_{\mathrm{i}, \mathrm{t}}^{\mathrm{GOV}}$} & 1,62069 & 1,526313 & 1,71756 & 1,624274 \\
\hline & $(0,000)^{* * *}$ & $(0,000)^{* * *}$ & $(0,000)^{* * *}$ & $(0,000)^{* * *}$ \\
\hline \multirow{2}{*}{ SEM } & & & $-0,56399$ & 2,323807 \\
\hline & & & $(0,839)$ & $(0,476)$ \\
\hline \multirow{2}{*}{$\operatorname{car}_{\mathrm{i}, \mathrm{t}}{ }^{\mathrm{DO}} \mathrm{x}$ SEM } & & & $-0,16915$ & $-0,2349845$ \\
\hline & & & $(0,634)$ & $(0,538)$ \\
\hline \multirow{2}{*}{$\operatorname{car}_{i, t}{ }^{\mathrm{DV}}$ x SEM } & & & $-0,33004$ & $-0,3360049$ \\
\hline & & & $(0,002)^{* * *}$ & $(0,004)^{* * *}$ \\
\hline \multirow{2}{*}{$\operatorname{car}_{i, t}{ }^{G O V}$ x SEM } & & & $-1,55438$ & $-1,506754$ \\
\hline & & & $(0,001)^{* * *}$ & $(0,003)^{* * *}$ \\
\hline \multirow{2}{*}{$\mathrm{Vm}$} & & $-1,401348$ & & $-1,453078$ \\
\hline & & $(0,000)^{* * *}$ & & $(0,000) * * *$ \\
\hline \multirow{2}{*}{ Roa } & & $-0,0059985$ & & $-0,0056158$ \\
\hline & & $(0,762)$ & & $(0,776)$ \\
\hline \multirow{2}{*}{ Btm } & & $-0,9030349$ & & $-0,8896751$ \\
\hline & & $(0,000)^{* * *}$ & & $(0,000)^{* * *}$ \\
\hline \multirow{2}{*}{ IFRS } & & $-7,878638$ & & $-7,932209$ \\
\hline & & $(0,000)^{* * *}$ & & $(0,000)^{* * *}$ \\
\hline $\mathrm{R}^{2}$ ajustado & 0,2161 & 0,2325 & 0,2206 & 0,2368 \\
\hline Observações & 3514 & 2859 & 3514 & 2859 \\
\hline
\end{tabular}

Fonte: dados da pesquisa.

Nota: Em que $C A R_{i, t}$ é o retorno anormal acumulado do ativo $i$, no ano $t$, car ${ }_{i, t}^{D O}$ é o retorno anormal acumulado de três dias com centro nos anúncios de disclosure obrigatório das demonstrações contábeis, car $_{i, t}{ }^{D V}$ é o retorno anormal acumulado de três dias com centro nos anúncios de disclosure voluntário de comunicados ao mercado, $c a{ }_{i, t}{ }^{G O V}$ é o retorno anormal acumulado de três dias com centro nos anúncios com transferência informacional de classificação de rating soberano do governo, SEM é uma variável dummy assumindo valor 0 se a empresa não é SEM e 1 se a empresa é SEM, Vm é o logaritmo neperiano do valor de mercado das empresas, Roa é o retorno sobre o ativo, Btm é o Bookto-Market e IFRS é uma dummy assumindo 1 no ano de 2010 e nos anos posteriores e 0 caso contrário. Nos casos em que não tenha ocorrido anúncio, as variáveis $c a r_{i, t}{ }^{D O}, c_{i, t}{ }^{D V}$ e $c a r_{i, t}{ }^{G O V}$ assumiram o valor 0 e nos casos que ocorreram mais de um evento, os retornos foram somados. *** significante a $1 \%$.

Analisando o modelo 1, na Tabela 4, pode-se verificar que tanto a variável de evidenciação obrigatória, quanto as de evidenciação voluntária e de classificação de rating do governo apresentam significância ao nível de $1 \%$. Este resultado demonstra que essas variáveis têm impacto na variável dependente que é o retorno anormal acumulado anual. Verifica-se que as variáveis $V m$ e $B t m$, também, apresentaram significância. Tais resultados estão em linha com as hipóteses 1, 2 e 3a apresentadas nessa pesquisa.

No modelo 3, pode-se verificar que da mesma forma que nos modelos 1 e 2 as variáveis de evidenciação obrigatória, voluntária e de classificação de rating do governo apresentam significância ao nível de 1\%. Nas variáveis de interações não há significância para a interação da variável $S E M$ com a variável de evidenciação obrigatória, porém, há na interação com as variáveis de divulgação voluntária e de classificação de rating soberano. Este resultado demonstra que há um impacto adicional para as SEM em eventos de divulgação voluntária e de classificação de rating soberano. Tal fato mostra evidência na direção da hipótese $\mathrm{H}_{3 \mathrm{~b}}$ apresentada nesse estudo, ou seja, que as empresas de economia mista são afetadas de forma diferente no momento da divulgação do rating soberano quando comparadas com as demais empresas. Nos modelos 2 e 4 as variáveis de controle Vm, Btm e IFRS também apresentaram significância. 
Como robustez, foram realizados testes apenas com as empresas do setor de Finanças e Seguros, setor que mais contém SEM, e excluído esse setor. Todos os detalhes metodológicos utilizados nas estimações anteriores foram mantidos. Pode-se verificar que os resultados permaneceram semelhantes tanto para o setor de Finanças e Seguros quanto excluído esse setor.

Em resumo, em todos os modelos as variáveis de retornos anormais acumulados em torno das divulgações obrigatórias, voluntárias e de classificação de rating do governo apresentam impacto significativo nos retornos anormais acumulados anuais. Além disso, em todos os modelos com interações, as interações da variável SEM com a variável de evidenciação voluntária e de classificação de rating soberano apresentaram resultados significativos. E as variáveis $V m$, Btm e IFRS foram significativas nos modelos com controles.

Esses resultados corroboram pesquisas como a de Malaquias e Lemes (2015) ao encontrar impacto para evidenciação obrigatória, mas são contrários às pesquisas de Mugaloglu e Erdag (2011), Sadeghzadeh e Karimi (2010) e Marques et al. (2010), que não verificaram esse impacto. Quanto à evidenciação voluntária, os resultados se apoiam nas pesquisas de Murcia et al. (2011), Souza et al. (2014) e Lima et al. (2012). Os resultados confirmam ainda as pesquisas de Murcia, Murcia e Borba (2013), Creighton, Gower e Richards (2007) e Jorion, Liu e Shi (2005) ao relacionar valor e classificações de rating.

\section{CONSIDERAÇÕES FINAIS}

Esta pesquisa teve como objetivo verificar se a divulgação de informações impacta no valor das ações das empresas. De forma específica, se a divulgação de informações obrigatórias, por meio da publicação de demonstrações contábeis, voluntárias, por meio da publicação de comunicados ao mercado, e do governo, por meio da classificação de rating do governo afetam o valor das ações das sociedades anônimas e o impacto adicional nas sociedades de economia mista.

Para isso foi realizado um estudo semelhante ao de Beyer et al. (2010) para verificar se a divulgação de informações impacta o valor das ações das empresas. Diante dos resultados apresentados nos testes estatísticos, todas as hipóteses levantadas no estudo foram confirmadas. Assim, pode-se concluir que os investidores reagem à divulgação de demonstrações financeiras, comunicados ao mercado e de classificações de rating do governo, além de reagirem adicionalmente às divulgações voluntárias e de classificação de rating do governo quando se trata de sociedades de economia mista no mercado brasileiro.

A pesquisa contribui para uma melhor compreensão do mercado de capitais brasileiro e do comportamento dos investidores, mostrando que os meios de divulgação de informações estudados têm relevância e funcionam como redutores de assimetria informacional. O estudo contribui, também, com a literatura de disclosure e de transferência informacional, corroborando com alguns estudos e avançando na relação entre divulgação de informações e variáveis financeiras, além de destacar novas possibilidades de pesquisa.

Este estudo apresenta algumas limitações: o campo de estudo, que abrange apenas as empresas listadas na B3; os tipos de informações utilizadas como eventos, apenas a divulgação das demonstrações contábeis, dos comunicados ao mercado e de rating soberano; a forma de mensuração dos retornos anormais, que dentre tantos foi utilizado o modelo de retorno ajustado ao mercado; e a subjetividade das janelas de evento.

Como sugestões futuras recomenda-se a utilização de outros campos de estudo, por exemplo, segmentando as empresas por setor. Além disso, outros tipos de informação podem ser destacados, como fatos relevantes e notícias de mídia. Os estudos de eventos permitem também que sejam utilizados diversos métodos de mensuração dos retornos anormais, portanto, é possível realizar esta pesquisa utilizando outros métodos e com outras janelas de evento.

\section{REFERÊNCIAS}

Beyer, A., Cohen, D. A., Lys, T. Z., \& Walther, B. R. (2010). The financial reporting environment: Review of the recent literature. Journal of accounting and economics, 50(2), 296-343, 2010. DOI: https://doi.org/10.1016/j. jacceco.2010.10.003

Brasil. Lei das sociedades por ações: Lei $n{ }^{\circ}$ 6404, de 15 de dezembro de 1976. Diário Oficial da União, Brasília, DF, 15 de dezembro de 1976. Recuperado em 17 agosto, 2016 de http://www.planalto.gov.br/ccivil_03/leis/ L6404consol.htm. 
Camargos, M. A. D., \& Barbosa, F. V. (2003). Estudos de evento: teoria e operacionalização. Caderno de Pesquisas em Administração, São Paulo, 10(3), 01-20.

Comissão De Valores Mobiliários. Instrução CVM nº 247, 27 de março de 1996. Recuperado em 17 agosto, 2016, de http://www.cvm.gov.br/legislacao/inst/inst247.html.

Comitê De Orientação Para Divulgação De Informações Ao Mercado. Pronunciamento De Orientação $N^{\circ} 05$, de 27 de Novembro de 2008. Recuperado em 11 agosto, 2015, de http://www.codim.org.br/downloads/ Pronunciamento_Orientacao_05.pdf.

Creighton, A., Gower, L., \& Richards, A. J. (2007). The impact of rating changes in Australian financial markets. Pacific-Basin Finance Journal, 15(1), 1-17. DOI: https://doi.org/10.1016/j.pacfin.2006.04.003

Dietrich, J. R. (1989). Discussion of voluntary disclosure choice and earnings information transfer. Journal of Accounting Research, 106-110. DOI: https://doi.org/10.2307/2491067

Dye, R. A. (2001). An evaluation of 'essays on disclosure' and the disclosure literature in accounting. Journal of Accounting and Economics, 32(1-3), 181-235. DOI: https://doi.org/10.1016/S0165-4101(01)00024-6

Foster, G. (1981). Intra-industry information transfers associated with earnings releases. Journal of accounting and economics, 3(3), 201-232. DOI: https://doi.org/10.1016/0165-4101(81)90003-3

Gibbins, M., Richardson, A., \& Waterhouse, J. (1990). The management of corporate financial disclosure: opportunism, ritualism, policies, and processes. Journal of accounting research, 28(1), 121-143. DOI: https:// doi.org/10.2307/2491219

Healy, P. M., \& Palepu, K. G. (2001). Information asymmetry, corporate disclosure, and the capital markets: A review of the empirical disclosure literature. Journal of accounting and economics, 31(1), 405-440. DOI: https://doi.org/10.1016/S0165-4101(01)00018-0

Jorion, P., Liu, Z., \& Shi, C. (2005). Informational effects of regulation FD: evidence from rating agencies. Journal of financial economics, 76(2), 309-330. DOI: https://doi.org/10.1016/j.jfineco.2004.05.001

Kusch B., N., Oliveira Camilo, S. P., Marcon, R., Bandeira-De-Mello, R. (2014). Conexões políticas em estruturas de propriedade: o governo como acionista em uma análise descritiva. Revista de Administração Mackenzie, 15(1), 98-124. DOI: https://doi.org/10.1590/S1678-69712014000100005

Leuz, C., \& Wysocki, P. D. (2008). Economic consequences of financial reporting and disclosure regulation: a review and suggestions for future research. DOI: https://doi.org/10.2139/ssrn.1105398

Lima, D. H. S., Rodrigues, J. M., Silva, C. A. T., \& Silva, J. D. G. (2012). Impacto do Nível de Evidenciação de Informações Contábeis sobre a Precificação de Ações no Contexto de Seleção Adversa: uma pesquisa experimental. Revista Brasileira de Gestão de Negócios, 14(43), 159-175.

Malaquias, R. F., \& Lemes, S. (2015). Evidenciação e Volatilidade: Testes com Equações Estruturais. BASERevista de Administração e Contabilidade da Unisinos, 12(2), 96-109. DOI: https://doi.org/10.4013/ base.2015.122.02

Marques, V. A., Lara, C. O., Ayala, L. C., \& Lamounier, W. M. (2010). O Impacto dos Fatos Relevantes no Mercado de Capitais: um estudo dos fatos publicados pelas empresas participantes do Novo Mercado do segmento Bovespa. Sociedade, Contabilidade e Gestão, 5(3).

Mugaloglu, Y. I., \& Erdag, E. (2011). The Relationship between Stock Return Volatility and Public Disclosure: The Case of Istanbul Stock Exchange Online Public Disclosure Platform. Journal of Applied Economics \& Business Research, 1(2), 93-102.

Murcia, F. D., Souza, F. C., Dill, R. P., \& Costa Jr, N. C. A. (2011). Impacto do nível de disclosure corporativo na volatilidade das ações de companhias abertas no Brasil. Revista de Economia e Administração, 10(2), 196218.

Ribeiro, M. C. P., \& Alves, R. S. (2006). Sociedades estatais, controle e lucro. Scientia Iuris, 10, 163-182. DOI: https://doi.org/10.5433/2178-8189.2006v10n0p163

Sadeghzadeh, A., \& Karimi, J. (2010). Determining of relationship between information disclosures and price fluctuations of acceptrd companies in Tehran Stock Exchange. International Journal of Academic Research, 2(5), 351-355. 
Salotti, B. M., \& Yamamoto, M. M. (2005). Ensaio sobre a teoria da divulgação. BBR-Brazilian Business Review, 2(1), 53-70. DOI: https://doi.org/10.15728/bbr.2005.2.1.4

Schneible, R. A. (2015). Information transfer and firm-level strategy. Accounting \& Finance, 56(4), 1119-1148. DOI: https://doi.org/10.1111/acfi.12112

Skillius, A., \& Wennberg, U. (1998). Continuity, credibility and comparability: key challenges for corporate environmental performance measurement and communication. The international Institute for Industrial Environmental Economics at Lund University. Lund. Disponível em: www.eea.europa.eu/publications/ESS09.

Sousa, C. B., Silva, A. F., Ribeiro, M. S., \& Weffort, E. F. J. (2014). Valor de mercado e disclosure voluntário: Estudo empírico em companhias listadas na BM\&FBovespa. Revista Ambiente Contábil, 6(2), 94-115.

Souza Murcia, F. C., Murcia, F. D., \& Borba, J. A. (2013). The Informational Content of Credit Ratings in Brazil: An Event Study. Brazilian Review of Finance, 11(4), 503-526.

Vasconcelos Rocha Mapurunga, P., Rodrigues Ponte, V. M., \& Pinheiro Holanda, A. (2014). Fatores explicativos da aderência das firmas brasileiras ao disclosure relativo às informações por segmento. Enfoque: Reflexão Contábil, 33(1), 53-69. DOI: https://doi.org/10.4025/enfoque.v33i1.20743

Verrecchia, R. E. (2001). Essays on disclosure. Journal of Accounting and Economics, 32(1-3), 97-180. DOI: https://doi.org/10.1016/S0165-4101(01)00025-8

Von Alberti-Alhtaybat, L., Hutaibat, K., \& Al-Htaybat, K. (2012). Mapping corporate disclosure theories. Journal of Financial Reporting and Accounting, 10(1), 73-94. DOI: https://doi.org/10.1108/19852511211237453

\section{Como citar este artigo}

Locatelli, O.; Nossa, V.; \& Ferreira, F. R. (2020). Impacto da evidenciação de informações no valor das ações das sociedades de economia mista. Revista de Contabilidade e Organizações, 14:e168631. DOI: http://dx.doi.org/10.11606/issn.1982-6486.rco.2020.168631 